\title{
A new note on absolute matrix summability
}

\section{Hikmet S Özarslan* and Enes Yavuz}

${ }^{*}$ Correspondence:

seyhan@erciyes.edu.tr

Department of Mathematics, Erciyes

University, Kayseri, 38039, Turkey

\section{Abstract}

In the present paper, we have proved theorems dealing with matrix summability factors by using quasi $\beta$-power increasing sequences. Some new results have also been obtained.

MSC: 40D15; 40F05; 40G99

Keywords: absolute matrix summability; quasi power increasing sequences; infinite series

\section{Introduction}

A positive sequence $\left(\gamma_{n}\right)$ is said to be quasi $\beta$-power increasing sequence if there exists a constant $K=K(\beta, \gamma) \geq 1$ such that $K n^{\beta} \gamma_{n} \geq m^{\beta} \gamma_{m}$ holds for all $n \geq m \geq 1$ [1]. A sequence $\left(\lambda_{n}\right)$ is said to be of bounded variation, denote by $\left(\lambda_{n}\right) \in \mathcal{B V}$, if $\sum_{n=1}^{\infty}\left|\Delta \lambda_{n}\right|=$ $\sum_{n=1}^{\infty}\left|\lambda_{n}-\lambda_{n+1}\right|<\infty$. Let $\sum a_{n}$ be a given infinite series with the partial sums $\left(s_{n}\right)$. Let $\left(p_{n}\right)$ be a sequence of positive numbers such that

$$
P_{n}=\sum_{v=0}^{n} p_{v} \rightarrow \infty \quad \text { as } n \rightarrow \infty\left(P_{-i}=p_{-i}=0, i \geq 1\right)
$$

The sequence-to-sequence transformation

$$
\sigma_{n}=\frac{1}{P_{n}} \sum_{v=0}^{n} p_{\nu} s_{v}
$$

defines the sequence $\left(\sigma_{n}\right)$ of the $\left(\bar{N}, p_{n}\right)$ mean of the sequence $\left(s_{n}\right)$, generated by the sequence of coefficients $\left(p_{n}\right)$ [2].

The series $\sum a_{n}$ is said to be summable $\left|\bar{N}, p_{n}\right|_{k}, k \geq 1$ if [3]

$$
\sum_{n=1}^{\infty}\left(\frac{P_{n}}{p_{n}}\right)^{k-1}\left|\sigma_{n}-\sigma_{n-1}\right|^{k}<\infty
$$

Let $A=\left(a_{n v}\right)$ be a normal matrix, i.e., a lower triangular matrix of nonzero diagonal entries. Then $A$ defines the sequence-to-sequence transformation, mapping the sequence $s=\left(s_{n}\right)$ to $A s=\left(A_{n}(s)\right)$, where

$$
A_{n}(s)=\sum_{v=0}^{n} a_{n v} s_{v}, \quad n=0,1, \ldots
$$


The series $\sum a_{n}$ is said to be summable $\left|A, p_{n}\right|_{k}, k \geq 1$ if [4]

$$
\sum_{n=1}^{\infty}\left(\frac{P_{n}}{p_{n}}\right)^{k-1}\left|\bar{\Delta} A_{n}(s)\right|^{k}<\infty,
$$

where

$$
\bar{\Delta} A_{n}(s)=A_{n}(s)-A_{n-1}(s) .
$$

Before stating the main theorem, we must first introduce some further notations.

Given a normal matrix $A=\left(a_{n v}\right)$, we associate two lower semimatrices $\bar{A}=\left(\bar{a}_{n v}\right)$ and $\hat{A}=\left(\hat{a}_{n v}\right)$ as follows:

$$
\bar{a}_{n v}=\sum_{i=v}^{n} a_{n i}, \quad n, v=0,1, \ldots
$$

and

$$
\hat{a}_{00}=\bar{a}_{00}=a_{00}, \quad \hat{a}_{n v}=\bar{a}_{n v}-\bar{a}_{n-1, v}, \quad n=1,2, \ldots
$$

It may be noted that $\bar{A}$ and $\hat{A}$ are the well-known matrices of series-to-sequence and seriesto-series transformations, respectively. Then, we have

$$
A_{n}(s)=\sum_{v=0}^{n} a_{n v} s_{v}=\sum_{v=0}^{n} \bar{a}_{n v} a_{v}
$$

and

$$
\bar{\Delta} A_{n}(s)=\sum_{\nu=0}^{n} \hat{a}_{n v} a_{v} .
$$

\section{Known result}

Recently, many authors have come up with theorems dealing with the applications of power increasing sequences $[1,5-7]$. Among them, Bor and Özarslan have proved two theorems for $\left|\bar{N}, p_{n}\right|_{k}$ summability method by using quasi $\beta$-power increasing sequence [5]. Their theorems are as follows.

Theorem A Let $\left(X_{n}\right)$ be a quasi $\beta$-power increasing sequence for some $0<\beta<1$, and let there be sequences $\left(\beta_{n}\right)$ and $\left(\lambda_{n}\right)$ such that

$$
\begin{aligned}
& \left|\Delta \lambda_{n}\right| \leq \beta_{n}, \\
& \beta_{n} \rightarrow 0 \quad \text { as } n \rightarrow 0, \\
& \sum_{n=1}^{\infty} n\left|\Delta \beta_{n}\right| X_{n}<\infty, \\
& \left|\lambda_{n}\right| X_{n}=O(1) \text { as } n \rightarrow \infty .
\end{aligned}
$$


If

$$
\begin{aligned}
& \sum_{v=1}^{n} \frac{\left|s_{\nu}\right|^{k}}{v}=O\left(X_{n}\right), \\
& \sum_{n=1}^{m} \frac{p_{n}}{P_{n}}\left|s_{n}\right|^{k}=O\left(X_{m}\right), \quad m \rightarrow \infty,
\end{aligned}
$$

then $\sum a_{n} \lambda_{n}$ is summable $\left|\bar{N}, p_{n}\right|_{k}, k \geq 1$.

Theorem B Let $\left(X_{n}\right)$ be a quasi $\beta$-power increasing sequence for some $0<\beta<1$, and let sequences $\left(\beta_{n}\right)$ and $\left(\lambda_{n}\right)$ satisfy conditions (10)-(13) and (15). If

$$
\begin{aligned}
& \sum_{n=1}^{\infty} P_{n}\left|\Delta \beta_{n}\right| X_{n}<\infty, \\
& \sum_{n=1}^{m} \frac{\left|s_{n}\right|^{k}}{P_{n}}=O\left(X_{m}\right),
\end{aligned}
$$

then $\sum a_{n} \lambda_{n}$ is summable $\left|\bar{N}, p_{n}\right|_{k}, k \geq 1$.

\section{The main result}

The aim of this paper is to generalize Theorem $\mathrm{A}$ and Theorem $\mathrm{B}$ to $\left|A, p_{n}\right|_{k}$ summability. Now, we shall prove the following two theorems.

Theorem 1 Let $A=\left(a_{n v}\right)$ be a positive normal matrix such that

$$
\begin{aligned}
& \bar{a}_{n 0}=1, \quad n=0,1, \ldots, \\
& a_{n-1, v} \geq a_{n v}, \quad \text { for } n \geq v+1, \\
& a_{n n}=O\left(\frac{p_{n}}{P_{n}}\right),
\end{aligned}
$$

and $\left(X_{n}\right)$ is a quasi $\beta$-power increasing sequence for some $0<\beta<1$. If all the conditions of Theorem $\mathrm{A}$ and

$$
\left(\lambda_{n}\right) \in \mathcal{B V}
$$

are satisfied, then the series $\sum a_{n} \lambda_{n}$ is summable $\left|A, p_{n}\right|_{k}, k \geq 1$.

In the special case of $a_{n v}=\frac{p_{v}}{P_{n}}$, this theorem reduces to Theorem A.

Theorem 2 Let $A=\left(a_{n v}\right)$ be a positive normal matrix as in Theorem 1, and let $\left(X_{n}\right)$ is a quasi $\beta$-power increasing sequence for some $0<\beta<1$. If all the conditions of Theorem $\mathrm{B}$ and (21) are satisfied, then the series $\sum a_{n} \lambda_{n}$ is summable $\left|A, p_{n}\right|_{k}, k \geq 1$.

We need following lemmas for the proof of our theorems. 
Lemma 1 [1] Let $\left(X_{n}\right)$ be a quasi $\beta$-power increasing sequence for some $0<\beta<1$. If conditions (11) and (12) satisfied, then

$$
\begin{aligned}
& n X_{n} \beta_{n}=O(1) \quad \text { as } n \rightarrow \infty, \\
& \sum_{n=1}^{\infty} X_{n} \beta_{n}<\infty .
\end{aligned}
$$

Lemma 2 Let $\left(X_{n}\right)$ be a quasi $\beta$-power increasing sequence for some $0<\beta<1$. If conditions (11) and (16) are satisfied, then

$$
\begin{aligned}
& P_{n} \beta_{n} X_{n}=O(1), \\
& \sum_{n=1}^{\infty} p_{n} \beta_{n} X_{n}<\infty .
\end{aligned}
$$

The proof of Lemma 2 is similar to that of Bor in [8] and hence is omitted.

\section{Proof of Theorem 1}

Let $\left(T_{n}\right)$ denote A-transform of the series $\sum a_{n} \lambda_{n}$. Then by (8), (9) and applying Abel's transformation, we have

$$
\begin{aligned}
\bar{\Delta} T_{n} & =\sum_{v=1}^{n} \hat{a}_{n v} a_{v} \lambda_{v} \\
& =\sum_{v=1}^{n-1} \Delta_{v}\left(\hat{a}_{n v} \lambda_{v}\right) \sum_{k=1}^{v} a_{k}+\hat{a}_{n n} \lambda_{n} \sum_{v=1}^{n} a_{v} \\
& =\sum_{v=1}^{n-1}\left(\hat{a}_{n v} \lambda_{v}-\hat{a}_{n, v+1} \lambda_{v+1}\right) s_{v}+a_{n n} \lambda_{n} s_{n} \\
& =\sum_{v=1}^{n-1}\left(\hat{a}_{n v} \lambda_{v}-\hat{a}_{n, v+1} \lambda_{v+1}-\hat{a}_{n, v+1} \lambda_{v}+\hat{a}_{n, v+1} \lambda_{v}\right) s_{v}+a_{n n} \lambda_{n} s_{n} \\
& =\sum_{v=1}^{n-1} \Delta_{v}\left(\hat{a}_{n v}\right) \lambda_{v} s_{v}+\sum_{v=1}^{n-1} \hat{a}_{n, v+1} \Delta \lambda_{v} s_{v}+a_{n n} \lambda_{n} s_{n} \\
& =T_{n, 1}+T_{n, 2}+T_{n, 3} \quad \text { say. }
\end{aligned}
$$

Since

$$
\left|T_{n, 1}+T_{n, 2}+T_{n, 3}\right|^{k} \leq 3^{k}\left(\left|T_{n, 1}\right|^{k}+\left.T_{n, 2}\right|^{k}+\left.T_{n, 3}\right|^{k}\right),
$$

to complete the proof of the Theorem 1, it is sufficient to show that

$$
\sum_{n=1}^{\infty}\left(P_{n} / p_{n}\right)^{k-1}\left|T_{n, r}\right|^{k}<\infty, \quad \text { for } r=1,2,3
$$


First, applying Hölder's inequality with indices $k$ and $k^{\prime}$, where $k>1$ and $\frac{1}{k}+\frac{1}{k^{\prime}}=1$, we get that

$$
\begin{aligned}
\sum_{n=2}^{m+1}\left(\frac{P_{n}}{p_{n}}\right)^{k-1}\left|T_{n, 1}\right|^{k} & \leq \sum_{n=2}^{m+1}\left(\frac{P_{n}}{p_{n}}\right)^{k-1}\left(\sum_{v=1}^{n-1}\left|\Delta_{v} \hat{a}_{n v}\right|\left|\lambda_{v}\right|\left|s_{v}\right|\right)^{k} \\
& =O(1) \sum_{n=2}^{m+1}\left(\frac{P_{n}}{p_{n}}\right)^{k-1}\left(\sum_{v=1}^{n-1}\left|\Delta_{v} \hat{a}_{n v}\right|\left|\lambda_{v}\right|^{k}\left|s_{v}\right|^{k}\right) \times\left(\sum_{v=1}^{n-1}\left|\Delta_{v} \hat{a}_{n v}\right|\right)^{k-1} \\
& =O(1) \sum_{n=2}^{m+1}\left(\frac{P_{n}}{p_{n}} a_{n n}\right)^{k-1}\left(\sum_{v=1}^{n-1}\left|\Delta_{v} \hat{a}_{n v}\right|\left|\lambda_{v}\right|^{k}\left|s_{v}\right|^{k}\right) \\
& =O(1) \sum_{v=1}^{m}\left|\lambda_{v}\right|^{k}\left|s_{v}\right|^{k} \sum_{n=v+1}^{m+1}\left|\Delta_{v} \hat{a}_{n v}\right| \\
& =O(1) \sum_{v=1}^{m} \frac{p_{v}}{P_{v}}\left|\lambda_{v}\right|^{k-1}\left|\lambda_{v}\right|\left|s_{v}\right|^{k}=O(1) \sum_{v=1}^{m} \frac{p_{v}}{P_{v}}\left|\lambda_{v}\right|\left|s_{v}\right|^{k} \\
& =O(1) \sum_{v=1}^{m-1} \Delta\left|\lambda_{v}\right| \sum_{i=1}^{v} \frac{p_{i}}{P_{i}}\left|s_{i}\right|^{k}+O(1)\left|\lambda_{m}\right| \sum_{v=1}^{m} \frac{p_{v}}{P_{v}}\left|s_{v}\right|^{k} \\
& =O(1) \sum_{v=1}^{m-1} \beta_{v} X_{v}+O(1)\left|\lambda_{m}\right| X_{m} \\
& =O(1) \quad \text { as } m \rightarrow \infty
\end{aligned}
$$

by virtue of the hypotheses of Theorem 1 and Lemma 1.

Since $\left(\lambda_{n}\right) \in \mathcal{B V}$ by (21), applying Hölder's inequality with the same indices as those above, we have

$$
\begin{aligned}
\sum_{n=2}^{m+1}\left(\frac{P_{n}}{p_{n}}\right)^{k-1}\left|T_{n}(2)\right|^{k} \leq & \sum_{n=2}^{m+1}\left(\frac{P_{n}}{p_{n}}\right)^{k-1}\left(\sum_{v=1}^{n-1}\left|\Delta \lambda_{v}\right|\left|\hat{a}_{n, v+1}\right|\left|s_{v}\right|\right)^{k} \\
= & O(1) \sum_{n=2}^{m+1}\left(\frac{P_{n}}{p_{n}}\right)^{k-1}\left(\sum_{v=1}^{n-1}\left|\Delta \lambda_{v}\right|\left|\hat{a}_{n, v+1}\right|\left|s_{v}\right|^{k}\right) \\
& \times\left(\sum_{v=1}^{n-1}\left|\Delta \lambda_{v}\right|\left|\hat{a}_{n, v+1}\right|\right)^{k-1} \\
= & O(1) \sum_{n=2}^{m+1}\left(\frac{P_{n}}{p_{n}} a_{n n}\right)^{k-1}\left(\sum_{v=1}^{n-1} \beta_{v}\left|\hat{a}_{n, v+1}\right|\left|s_{v}\right|^{k}\right) \times\left(\sum_{v=1}^{n-1}\left|\Delta \lambda_{v}\right|\right)^{k-1} \\
= & O(1) \sum_{v=1}^{m} \beta_{v}\left|s_{v}\right|^{k} \sum_{n=v+1}^{m+1}\left|\hat{a}_{n, v+1}\right| \\
= & O(1) \sum_{v=1}^{m} \beta_{v}\left|s_{v}\right|^{k} \\
= & O(1) \sum_{v=1}^{m}\left(v \beta_{v}\right)^{\left|s_{v}\right|^{k}}
\end{aligned}
$$




$$
\begin{aligned}
& =O(1) \sum_{v=1}^{m-1} \Delta\left(v \beta_{v}\right) \sum_{i=1}^{v} \frac{\left|s_{i}\right|^{k}}{i}+O(1) m \beta_{m} \sum_{v=1}^{m} \frac{\left|s_{v}\right|^{k}}{v} \\
& =O(1) \sum_{v=1}^{m-1} \Delta\left(v \beta_{v}\right) X_{v}+O(1) m \beta_{m} X_{m} \\
& =O(1) \sum_{v=1}^{m-1} v\left|\Delta \beta_{v}\right| X_{v}+O(1) \sum_{v=1}^{m-1} \beta_{v+1} X_{v+1}+O(1) m \beta_{m} X_{m} \\
& =O(1) \quad \text { as } m \rightarrow \infty,
\end{aligned}
$$

by virtue of the hypotheses of Theorem 1 and Lemma 1 .

Finally, by following the similar process as in $T_{n, 1}$, we have that

$$
\begin{aligned}
\sum_{n=1}^{m}\left(\frac{P_{n}}{p_{n}}\right)^{k-1}\left|T_{n}(3)\right|^{k} & \leq \sum_{n=1}^{m}\left(\frac{P_{n}}{p_{n}}\right)^{k-1}\left|a_{n n}\right|^{k}\left|\lambda_{n}\right|^{k}\left|s_{n}\right|^{k} \\
& =O(1) \sum_{n=1}^{m} \frac{p_{n}}{P_{n}}\left|\lambda_{n}\right|\left|s_{n}\right|^{k} \\
& =O(1) \quad \text { as } m \rightarrow \infty .
\end{aligned}
$$

So, we get

$$
\sum_{n=1}^{\infty}\left(P_{n} / p_{n}\right)^{k-1}\left|T_{n, r}\right|^{k}<\infty, \quad \text { for } r=1,2,3 .
$$

This completes the proof of Theorem 1.

\section{Proof of Theorem 2}

Using Lemma 2 and proceeding as in the proof of Theorem 1, replacing $\sum_{v=1}^{m} \beta_{v}\left|s_{v}\right|^{k}$ by $\sum_{v=1}^{m} \beta_{v} P_{v}\left(\frac{\left|s_{v}\right|^{k}}{P_{v}}\right)$, we can easily prove Theorem 2 .

If we take $p_{n}=1$ in these theorems, then we have two new results dealing with $|A|_{k}$ summability factors of infinite series. Also, if we take $k=1$, then we obtain another two new results concerning $|A|$ summability. Finally, by taking $\left(X_{n}\right)$ as almost increasing sequence in the theorems, we get new results dealing with $\left|A, p_{n}\right|_{k}$ summability factors of infinite series.

\section{Competing interests}

The authors declare that they have no competing interests.

\section{Authors' contributions}

All authors contributed equally to the manuscript and read and approved the final manuscript.

Received: 21 November 2012 Accepted: 23 September 2013 Published: 07 Nov 2013

\section{References}

1. Leindler, L: A new application of quasi power increasing sequences. Publ. Math. (Debr.) 58, 791-796 (2001)

2. Hardy, GH: Divergent Series. Oxford University Press, Oxford (1949)

3. Bor, H: On two summability methods. Math. Proc. Camb. Philos. Soc. 97(1), 147-149 (1985)

4. Sulaiman, WT: Inclusion theorems for absolute matrix summability methods of an infinite series (IV). Indian J. Pure Appl. Math. 34(11), 1547-1557 (2003)

5. Bor, H, Özarslan, HS: On the quasi power increasing sequences. J. Math. Anal. Appl. 276, $924-929$ (2002)

6. Bor, H, Debnath, L: Quasi $\beta$-power increasing sequences. Int. J. Math. Math. Sci. 44, 2371-2376 (2004)

7. Bor, H: A general note on increasing sequences. J. Inequal. Pure Appl. Math. 8/3, 1-7 (2007)

8. Bor, H: On absolute summability factors for $\left|\bar{N}, p_{n}\right|_{k}$ summability. Comment. Math. Univ. Carol. 32/3, 435-439 (1991) 
10.1186/1029-242X-2013-474

Cite this article as: Özarslan and Yavuz: A new note on absolute matrix summability. Journal of Inequalities and Applications 2013, 2013:474

Submit your manuscript to a SpringerOpen ${ }^{\circ}$ journal and benefit from:

- Convenient online submission

- Rigorous peer review

- Immediate publication on acceptance

Open access: articles freely available online

- High visibility within the field

- Retaining the copyright to your article

Submit your next manuscript at $\gg$ springeropen.com 